\title{
Competencias clave y patrimonio en educación primaria. Un análisis de la concepción del profesorado en formación
}

\author{
Juan Ramón MORENO-VERA \\ Santiago PONSODA-LÓPEZ DE ATALAYA
}

Datos de contacto:

Juan Ramón Moreno-Vera Universidad de Murcia ir.moreno@um.es

Santiago Ponsoda-López de Atalaya

Universidad de Alicante santiago.ponsoda@ua.es

Recibido: 06/09/2021

Aceptado: 30/11/2021

\begin{abstract}
RESUMEN
La investigación que se presenta tiene como objetivo principal el análisis de las concepciones de los futuros docentes de Educación Primaria sobre la utilidad de la educación patrimonial a la hora de trabajar las competencias clave propuestas por el currículum. Para ello se diseñó, validó e implementó un cuestionario (escala Likert) entre 279 futuros maestros/as del grado de Maestro/a en Educación Primaria de la Universidad de Alicante cuyos resultados fueron analizados a través del paquete estadístico SPSS v24. Los resultados muestran cómo el alumnado considera el patrimonio un recurso válido para el trabajo de las diferentes competencias clave en el aula de Educación Primaria, si bien los futuros profesores lo consideran más adecuado para trabajar la conciencia y expresiones culturales y las competencias sociales y cívicas, mientras que el resto de competencias obtienen menores puntuaciones lo que indica, a modo de conclusión, que no se trabaja el patrimonio en un sentido holístico y transversal. Estos resultados ponen de manifiesto, por tanto, la necesidad de abordar la enseñanza a través del patrimonio desde un prisma amplio que tenga en cuenta algunas de sus principales potencialidades como es la transversalidad y la interdisciplinariedad, lo cual lo convertiría en un recurso de primer orden para abordar el tratamiento de las diferentes competencias claves.
\end{abstract}

PALABRAS CLAVE: Educación Primaria; patrimonio; concepciones; enseñanza-aprendizaje; competencias. 


\title{
Heritage and key competences in Primary Education. Analysis of teacher-in-training perceptions
}

\begin{abstract}
The main target in this investigation is to analyze the conception that future teachers of Primary Education have about the utility of heritage education to work the key competences proposed by the official curriculum. The reach that goal, a questionnaire (Likert scale 1-5) was designed, validated and implemented among 279 future teachers of Primary Education, now studying the Primary Education Degree in the University of Alicante (Spain). The analysis and procedural were carried by the SPSS v. 24 statistical package. The results show that future teachers consider heritage a valid resource to work the key competences in classroom, although it is important to note that, for the participants, is particularly adequate to work the competence of awareness and cultural expressions or social and civic competences. Instead of that, the rest of key competences obtained lower results, which indicate that future teachers do not consider heritage in a holistic and transversal way. These results show up, in conclusion, the need of teaching heritage education with wider approaches, taking in account its main potentialities, as transversality and interdisciplinarity, what could make heritage a better resource to work on the different key competences.
\end{abstract}

KEYWORDS: Primary education; heritage; conceptions; teaching-learning; competences.

\section{Introducción}

En el año 2006 en España se incorporaron las competencias clave (entonces denominadas "competencias básicas") a la legislación educativa española, siendo desde entonces, uno de los elementos centrales de la estructura curricular a nivel europeo (Egido, 2011). De hecho, la propia Comisión Europea las definía en 2004 como "conjunto de conocimientos y destrezas que los individuos necesitan para el desarrollo personal y que deben ser desarrolladas al acabar la enseñanza obligatoria" (Bolívar, 2010, p. 2).

Así pues, se desprende de tal definición que suponen una serie de conocimientos que serán desarrollados a lo largo de todas las etapas educativas y transversalmente mediante todas las áreas de conocimiento.

Sin embargo, estudios específicos como los de López-Facal et al. (2017) sobre la manera en la que las competencias clave eran introducidas en nuestras aulas, ya remarcaban que unas materias concretas están estrechamente relacionadas con sus correspondientes competencias concretas, dejando de lado el trabajo transversal y holístico de todas en su conjunto. De hecho, como señalan autores como RodríguezGarcía et al. (2017), el desarrollo de las competencias desde un punto de vista "amplio" sigue siendo una necesidad para la educación del siglo XXI. 
La relación entre la educación patrimonial y el desarrollo de las competencias clave aún presenta lagunas a nivel investigador, pese a que el propio currículo educativo reconoce que el patrimonio tiene un carácter transversal, lo que podría ayudar a ser un buen agente en la adquisición de las mismas (Real Decreto 126/2014).

En ese sentido, Fernández (2005) contempla el carácter social y transversal del patrimonio al afirmar "permite poner claridad en muchas materias y aporta conocimientos transversales [...] que apoyan la conformación de destrezas, valores y actitudes". Así mismo, García-Ceballos (2014) remarca la capacidad que tiene el patrimonio para trabajar la inclusión y su carácter social.

Sin embargo, para Salido-López (2017) la propia legislación pone en relación la educación artística (Fontal, 2016; Fontal et al., 2015) y patrimonial con la competencia en conciencia y expresiones culturales que, a su juicio, guarda relación con la Educación artística y patrimonial, lo que conlleva una particular relación con las Ciencias Sociales. En este sentido, Arjones-Fernández (2016) ya indica que el valor del patrimonio es "intrínseco" a la educación en conciencia y expresiones culturales.

El hecho de que existan competencias clave directamente relacionadas con áreas de conocimiento cuando, a priori, deben tener un carácter transversal, ha suscitado un importante debate y controversia. Por ejemplo, Pérez et al. (2019) en el ámbito de la Educación Física, comentan que existen dos posturas contrapuestas. Por un lado, los docentes que opinan que las competencias clave no tienen un gran aporte emancipador a nivel de contenidos conceptuales, mientras que otros docentes consideran, como aspecto positivo, que producen una mejora educativa siempre y cuando el enfoque de su inclusión en el aula sea deductivo e interdisciplinar.

De hecho, autores como Bolívar (2015) comentan que una de las principales ventajas de la inclusión en el currículum de las competencias clave desde el ámbito europeo, es que ofrecen la posibilidad de "superar las miradas e intereses particulares" $(2015,1)$ a la hora de realizar nuevas reformas educativas ya que permiten articular un amplio consenso curricular y estructurar los contenidos a través de competencias transversales.

Este carácter transversal como elemento nuclear del currículum educativo fue defendido por otros autores como Egido (2011) o Rodríguez-García et al. (2011) sin que, sin embargo, se haya conseguido dejar atrás una visión parcial y poco holística de las competencias clave que siguen muy ligadas a las áreas de conocimiento en las que se estructura el currículum español. Así, para Piñana (2019) lo que se necesita, en el caso español, es promover desde el currículum, un enfoque competencial que permita transformar la acción docente y la innovación en el aula dentro de un marco metodológico que se centre en la implementación de las competencias clave.

De este modo, la falta de transversalidad en el uso de las competencias clave, la no utilización de un enfoque competencial que organice los conocimientos en función de las competencias, así como el poco sentido holístico en el uso de las competencias se confirma en estudios como el de Cerezo et al. (2020) que han analizado, en el Decreto de educación primaria de la Comunitat Valenciana la frecuencia en la que cada una de las competencias clave se trabaja en los seis cursos del programa educativo oficial. La conclusión es clara ya que se apunta hacia "una falta de transversalidad en algunas 
competencias" (2020, 35).

Esto, según Cerezo et al. (2020) nos conduce hacia una necesidad de contar con un modelo competencial, así como una mayor interconexión entre los contenidos de las distintas materias.

En el caso de la enseñanza de las Ciencias Sociales, ya algunos autores como Pagès (2009) o De la Fuente (2011) indicaban la estrecha relación de la materia con las competencias sociales y cívicas. De hecho, De la Fuente (2011) proponía aplicar las competencias básicas en el área de Ciencias Sociales, supeditadas a los "valores éticos, morales y culturales" $(2011,36)$.

Pagès (2009) consideraba que la competencia social y ciudadana (nomenclatura que se recogía antes de la LOMCE, 2014, para las actuales competencias sociales y cívicas) era una competencia "a caballo" entre las más relacionadas con contenidos específicos de las áreas de conocimiento y las que tenían un carácter más transversal, por lo que ni siquiera reconocía en ella una competencia de carácter holístico que puede ser trabajada desde distintas materias escolares.

Estudios posteriores en el área de las Ciencias Sociales, como son los de GómezCarrasco (2013), Ull (2015) u Ortega y Gómez (2017) replanteaban la necesidad de acomodar los contenidos de Ciencias Sociales bajo un enfoque competencial para trabajarlos de manera transversal y no al revés. Gómez Carrasco (2013) abogaba por la necesidad de una evaluación ligada al desarrollo de competencias y no basada en la mera memorización de contenidos y conceptos de primer orden, tal y como se ha venido realizando tradicionalmente en la enseñanza de la historia, algo en lo que coincidía con los trabajos de Herrero y Pastor (2012) que también pensaban en modificar la evaluación en el título de Maestro de Educación Primaria acercándola hacia una evaluación competencial.

A su, vez, Ull (2015) realizaba una valiosa propuesta en el campo de las competencias sociales y cívicas al hablar de la necesidad de unas "competencias para la sostenibilidad" $(2015,46)$. Esta educación para la sostenibilidad, evidentemente, tienen un claro aporte interdisciplinar y transversal ya que soportaría los contenidos de disciplinas como la geografía, la economía, el derecho, la biología, la química o las ciencias ambientales. De hecho, en los últimos años, los problemas de cambio climático (Morote y Moreno-Vera, 2021) han aparecido como una preocupación en aumento para nuestra sociedad (a nivel ambiental, económico y turístico) y esto ha tenido una fuerte repercusión, primero en movimientos sociales ciudadanos (Fridays for Future) y, más tarde, a nivel escolar con la inclusión del cambio climático en los currículos oficiales.

También, los estudios de Ortega y Gómez (2017) han hecho hincapié en la necesidad de acomodar contenidos de Ciencias Sociales, tanto de historia, como de geografía al desarrollo de una competencia digital ligada al marco de aprendizaje TPACK (Koelher \& Mishra, 2008) que considera fundamental la correcta aplicación de contenidos específicos con plataformas y recursos digitales para que el aprendizaje del alumnado se produzca.

En los últimos años, investigaciones en didáctica de las Ciencias Sociales han seguido esta línea de potencial la enseñanza de las mismas vinculadas a las 
competencias digitales, incluso utilizando la enseñanza del patrimonio como recurso holístico que permita usar todas las competencias y no solo las directamente relacionadas con el área disciplinar. Así, el trabajo de Kortabitarte et al. (2018) se centra en el uso de una app para teléfonos inteligentes, como recurso para usar en un proyecto titulado "Arte medieval", en el que alumnado de 2ำ de Educación Secundaria tendrían que analizar, a través del patrimonio, la arquitectura románica y gótica.

Y, aunque el ámbito de la competencia digital, ha sido el que más estudios transversales ha posibilitado en el ámbito de las Ciencias Sociales y su enseñanza (Colomer et al., 2018), poco a poco van apareciendo nuevas líneas de investigación que permiten el análisis de la enseñanza de las competencias clave en Ciencias Sociales desde un punto de vista interdisciplinar más amplio y transversal. Así, Gómez Trigueros y Ruiz Bañuls (2017) comentaban la opción de enseñar por competencias en Ciencias Sociales mediante el Aprendizaje Basado en Proyectos (ABP), en su caso transversalmente trabajando contenidos de Ciencias Sociales y Literatura. Por su parte, Bustos et al. (2020) proponen el uso de la metodología del aprendizaje basado en retos (ABR) para el desarrollo en el aula de las competencias clave, algo que también sería posible mediante el uso del Aprendizaje-Servicio. También, Najarro y Maroto (2019), estudiaban como trabajar las competencias clave mediante salidas didácticas fuera del aula visitando el patrimonio fenicio de Almuñécar (Granada) o, Morote y Moreno-Vera (2021) que comentan la necesidad de incluir, de manera científica y rigurosa, los contenidos ligados a los problemas de cambio climático en la enseñanza de los Grados de Educación Primaria, para que los futuros ciudadanos reciban una enseñanza ambiental acorde a los problemas sociales actuales.

Además, el trabajo de Rodríguez-Lestegás et al. (2020) comenta la necesidad, de cara a las nuevas reformas legislativas del futuro, de centrar los esfuerzos tanto en el contenido conceptual como en el trabajo competencial, ya que es importante aprender transversalmente mediante los conocimientos que aportan las Ciencias Sociales.

En este sentido, la enseñanza y el aprendizaje de las Ciencias Sociales tienen la puerta abierta a trabajar sus contenidos transversalmente junto a los de otras disciplinas escolares, a pesar de las dificultades que estudios como los de VázquezCano (2016) evidencian en cuanto a la planificación docente por parte del profesorado. Esta visión de conjunto, más cerca del enfoque competencial, supone un reto de cara al futuro, para desarrollar un aprendizaje basado en el desarrollo de las competencias clave y no tanto en el tradicional desarrollo del contenido conceptual de cada área de conocimiento por separado que todavía hoy sigue predominando en las concepciones docentes sobre el aprendizaje de su alumnado (Restrepo, 2017) y que Sánchez (2017) considera "hegemónicas".

Así pues, este trabajo pretende analizar cuáles son las concepciones que tiene el futuro profesorado de Educación Primaria sobre cómo el patrimonio puede ayudar al alumnado a adquirir las diferentes competencias clave, ya que, según los antecedentes teóricos está estrechamente relacionado fundamentalmente con unas pocas competencias clave y eso limita su uso como recurso educativo para trabajar el resto, al no ser utilizado como un recurso didáctico transversal que permita un enfoque competencial de la educación. 


\section{Método}

\section{Objetivos específicos e hipótesis}

Tal y como se ha comentado al inicio de esta investigación, el objetivo principal de este estudio es el de analizar la concepción que tiene el futuro profesorado de Educación Primaria en relación al uso del patrimonio como recurso didáctico para desarrollar las competencias clave que establece el currículum oficial.

Para conseguir dicho objetivo se han considerado dos objetivos específicos:

- O.E. 1: Diseñar y validar un instrumento de evaluación "ad hoc" para medir el grado de percepción de los futuros docentes.

- O.E. 2: Analizar la concepción de los participantes en base a cada una de las siete competencias clave.

Estos objetivos específicos se sustentan en la hipótesis inicial de esta investigación que, basándose en otros estudios como los de López-Facal et al. (2017), establece que la educación patrimonial está estrechamente relacionada con las competencias sociales y cívicas, así como con la conciencia y expresión cultural pero que, presumiblemente, tendría menor relación con las otras cinco competencias clave.

\section{Participantes}

En lo que respecta a los participantes en el estudio, el cuestionario fue implementado durante el curso 2019/20 en la asignatura de "Didáctica de las Ciencias Sociales: historia" de 3o del Grado de Maestro/a en Educación Primaria de la Universidad de Alicante. Los participantes, estudiantes del grado y futuros docentes, fueron 279 personas de las que el $70^{\prime} 96 \%$ son mujeres ( $\mathrm{n}=198$ ) y un $29^{\prime} 04 \%$ hombres $(\mathrm{n}=81)$, siendo la media de edad de 21,39 años de edad.

\section{Instrumento de investigación}

Para cumplir con el primero de los objetivos específicos de esta investigación, se procedió a diseñar, validar e implementar un instrumento de evaluación consistente en un cuestionario con 7 ítems referidos al bloque de las competencias clave y su relación con la utilización del patrimonio como recurso educativo. El cuestionario fue diseñado "ad hoc" para esta investigación, aunque está basado en experiencias previas satisfactorias en el ámbito de la enseñanza de las Ciencias Sociales, como la de MorenoVera et al. (2020). Cada uno de los siete ítems se valoraba en una escala tipo Likert (15) siendo 1 el menor grado de acuerdo y 5 el mayor. Los ítems aparecidos en el instrumento se corresponden con cada una de las competencias clave establecidas por el Real Decreto 126/2014:

1. Trabajar con el Patrimonio permite al alumnado desarrollar la competencia matemática y en ciencia y tecnología en Primaria. 
2. Trabajar con el Patrimonio permite al alumnado desarrollar la competencia digital en Primaria.

3. Trabajar con el Patrimonio permite al alumnado desarrollar la competencia en conciencia y expresiones culturales en Primaria.

4. Trabajar con el Patrimonio permite al alumnado desarrollar la competencia de aprender a aprender en Primaria.

5. Trabajar con el Patrimonio permite al alumnado desarrollar las competencias sociales y cívicas en Primaria.

6. Trabajar con el Patrimonio permite al alumnado desarrollar la competencia en sentido de Iniciativa y espíritu emprendedor en Primaria.

7. Trabajar con el Patrimonio permite al alumnado desarrollar la competencia comunicación lingüística en Primaria.

\section{Procedimiento de análisis y fiabilidad del instrumento}

Esta investigación ha seguido un procedimiento de tipo cuantitativo a través del análisis estadístico-descriptivo de los datos mediante el paquete IBM SPSS v.25 que ha permitido realizar una interpretación de frecuencias y porcentajes, como ya se ha realizado en otros estudios del área de la enseñanza de las Ciencias Sociales como los de Gómez-Carrasco (2019; 2020).

Para establecer la fiabilidad del instrumento, en primer lugar, se realizó un análisis estadístico de los resultados medios y su correspondiente desviación estándar (SD) que se mantuvo siempre en valores aceptables entre 0 y 1 . En segundo lugar, y para medir la validez interna del propio constructo se estableció la prueba del Alfa de Cronbach ya que permite medir la consistencia interna de los ítems. Así, se obtuvo un resultado de 0.869 en el Alfa de Cronbach (Tabla 1), lo que según estudios como los de Getsdóttir et al. (2018), ofrece una muy alta consistencia interna en escalas bidimensionales al encontrarse entre los valores de 0.60 y .90 que sería lo aceptable para este tipo de estudios.

\section{Tabla 1}

Resultado del Alfa de Cronbach

\begin{tabular}{cc}
\hline \multicolumn{2}{c}{ Estadísticas de fiabilidad } \\
\hline Alfa de Cronbach & N de elementos \\
, 869 & 7 \\
\hline
\end{tabular}




\section{Resultados y discusión}

A continuación, serán analizados los resultados de la investigación relativos a los valores medios de cada uno de los 7 ítems que componían el instrumento.

\section{Tabla 2}

Análisis de media y desviación estándar

\begin{tabular}{lccccccc}
\hline \multicolumn{7}{c}{ Estadísticos } \\
\hline \multicolumn{1}{c}{ N válido } & 279 & 279 & 279 & 279 & 279 & 279 & 279 \\
\hline Media & 3,5269 & 3,7706 & 4,6344 & 4,4086 & 4,6237 & 3,9821 & 4,2115 \\
Mediana & 4,000 & 4,000 & 5,000 & 5,000 & 5,000 & 4,000 & 4,000 \\
Desviación estándar & 1,082408 & 0.97693 & 0,67512 & 0,84270 & 0,67718 & 0,98717 & 0,87022 \\
\hline
\end{tabular}

Según se puede observar en la Tabla 2 de las siete competencias clave analizadas en el estudio, hay 4 de ellas que tienen unos valores medios altos entre el 4 y el 5 , lo que supone que los futuros docentes de Educación Primaria sí que consideran que trabajar con el patrimonio ayudará a los estudiantes a trabajar esas competencias clave en concreto. Las cuatro respuestas positivas corresponden a las competencias de conciencia y expresiones culturales (4.63), competencias sociales y cívicas (4.63), competencia en aprender a aprender (4.40) y competencia lingüística (4.21).

Como se puede observar, los dos valores más altos corresponden a las competencias en conciencia y expresiones culturales y a las sociales y cívicas, lo que se corresponde con la hipótesis de inicio de la investigación y corrobora las teorías de López-Facal et al. (2017) y Arjones-Fernández (2016) sobre la estrecha relación que hay entre determinadas competencias clave y sus áreas de conocimiento afines.

Por el contrario, nos encontramos al mismo tiempo, con otras tres competencias que reciben una valoración media más baja entre los valores de 3 y 4 por lo que a pesar de no ser un resultado negative en absoluto, los futuros docentes presentan más dudas o desconfianza en que sean competencias clave que puedan ser trabajadas a través del patrimonio. Estas competencias son el sentido de la iniciativa y el espíritu emprendedor (3.98), la competencia digital (3.77) y, con el valor más bajo la competencia matemáticas y competencias básicas en ciencia y tecnología (3.52).

La concepción de los futuros docentes es que estas competencias tienen mayor relación con las áreas científico-técnicas que con las Ciencias Sociales (Restrepo, 2017) y las humanidades, por lo que el patrimonio no sería, en su opinión, el recurso más idóneo para trabajarlas, pese a que estudios como los de Cózar y Sáez (2017) unen contenidos de Ciencias Sociales con elementos como la realidad aumentada y las nuevas tecnologías o, incluso, existen estudios con buenos resultados que permiten la enseñanza en nuevas tecnologías usando como recursos elementos patrimoniales 
(Gómez-Redondo et al., 2016; Ibáñez-Etxeberría et al., 2018).

Si atendemos al análisis pormenorizado de cada una de las 7 competencias clave, se puede observar el grado de acuerdo o desacuerdo que mostraron los futuros docentes participantes en la investigación.

\section{Tabla 3}

Competencia matemática y competencias básicas en ciencia y tecnología

\begin{tabular}{ccccc}
\hline \multicolumn{5}{c}{ Variable 1 } \\
\hline 1 & Frecuencia & Porcentaje & Porcentaje válido & Porcentaje acumulado \\
\hline 2 & 9 & 3,2 & 3,2 & 3,2 \\
3 & 30 & 10,8 & 10,8 & 14,0 \\
4 & 99 & 35,5 & 35,5 & 49,5 \\
5 & 87 & 31,2 & 31,2 & 80,6 \\
Total & 54 & 19,4 & 19,4 & 100 \\
\hline
\end{tabular}

Al observar la Tabla 3, referida a la competencia matemática y las competencias básicas en ciencia y tecnología, vemos como un 14\% de la muestra está en desacuerdo o muy en desacuerdo de que se pueda trabajar a través del patrimonio, mientras que el resultado más alto es de un 35.5\% que responde indiferente (ni de acuerdo, ni en desacuerdo) a que sea el recurso óptimo para trabajar esta competencia.

\section{Tabla 4}

Competencia digital

Variable 2

\begin{tabular}{ccccc}
\hline & Frecuencia & Porcentaje & Porcentaje válido & Porcentaje acumulado \\
\hline 1 & 7 & 2,5 & 2,5 & 2,5 \\
2 & 14 & 5,0 & 5,0 & 7,5 \\
3 & 88 & 31,5 & 31,5 & 39,1 \\
4 & 97 & 34,8 & 34,8 & 73,8 \\
5 & 73 & 26,2 & 26,2 & 100 \\
Total & 279 & 100 & 100 & \\
\hline
\end{tabular}

Un resultado similar, aunque con una mayor valoración positiva, nos lo encontramos cuando observamos la Tabla 4 que se refiere a la competencia digital. En este caso solo un 7'5\% de estudiantes se muestran en desacuerdo, un 31,5\% responden 
de forma indiferente, mientras que el resultado más elevado corresponde al 34,8\% de los futuros docentes que sí se muestran de acuerdo, lo que coincide con los estudios mencionados que se centran en el uso de las TIC también para trabajar alrededor del patrimonio (Ibáñez-Etxeberría et al., 2018).

\section{Tabla 5}

Competencia en conciencia y expresiones culturales

\section{Variable 3}

\begin{tabular}{ccccc}
\hline & Frecuencia & Porcentaje & Porcentaje válido & Porcentaje acumulado \\
\hline 1 & 2 & 0,7 & 0,7 & 0,7 \\
2 & 3 & 1,1 & 1,1 & 1,8 \\
3 & 10 & 3,6 & 3,6 & 5,4 \\
4 & 65 & 23,3 & 23,3 & 28,7 \\
5 & 199 & 71,3 & 71,3 & 100 \\
Total & 279 & 100 & 100 & \\
\hline
\end{tabular}

En cuanto a los resultados que muestra la Tabla 5 sobre la competencia en conciencia y expresiones culturales, se puede observar un altísimo grado de acuerdo de los participantes en cuanto al trabajo de esta competencia mediante el patrimonio ya que destacan un $71,3 \%$ de la muestra que responde muy de acuerdo y un $23,4 \%$ que está de acuerdo lo que supone que el $94,6 \%$ de la muestra percibe positivamente la relación entre los recursos patrimoniales y el trabajo en esta competencia (López-Facal et al., 2017).

\section{Tabla 6}

Competencia de aprender a aprender

\section{Variable 4}

\begin{tabular}{ccccc}
\hline & Frecuencia & Porcentaje & Porcentaje válido & Porcentaje acumulado \\
\hline 1 & 5 & 1,8 & 1,8 & 1,8 \\
2 & 4 & 1,4 & 1,4 & 3,2 \\
3 & 23 & 8,2 & 8,2 & 11,5 \\
4 & 87 & 31,2 & 31,2 & 42,7 \\
5 & 160 & 57,3 & 57,3 & 100 \\
Total & 279 & 100 & 100 & \\
\hline
\end{tabular}

La Tabla 6, por su parte, también muestra unos resultados muy favorables al destacar un 57,3\% de la muestra muy de acuerdo y un 31,2\% de los futuros docentes 
que están de acuerdo. En este caso, la competencia de aprender a aprender, por su carácter completamente transversal puede ser trabajada por múltiples recursos educativos, uno de ellos, el patrimonio.

\section{Tabla 7}

Competencias sociales y cívicas

\section{Variable 5}

\begin{tabular}{ccccc}
\hline & Frecuencia & Porcentaje & Porcentaje válido & Porcentaje acumulado \\
\hline 1 & 3 & 1,1 & 1,1 & 1,1 \\
2 & 1 & 0,4 & 0,4 & 1,4 \\
3 & 10 & 3,6 & 3,6 & 5,0 \\
4 & 70 & 25,1 & 25,1 & 30,1 \\
5 & 195 & 69,9 & 69,9 & 100 \\
Total & 279 & 100 & 100 & \\
\hline
\end{tabular}

En la Tabla 7, relacionada con las competencias sociales y cívicas, es donde, de nuevo, encontramos los resultados más positivos de los todos los ítems. Destaca un $69,9 \%$ muy de acuerdo y un 25,1\% de acuerdo, lo que supone un $95 \%$ de los participantes que valoran positivamente trabajar esta competencia mediante la educación patrimonial. Esta buena percepción se relaciona con los estudios de Cuenca et al. (2017) o González-Monfort (2019) que ya indican la importancia de la educación patrimonial en la conformación de una buena identidad democrática, cívica y ciudadana. De hecho, incluso el currículum oficial (Moreno-Vera \& Alvén, 2020), señala la necesidad de trabajar mediante la educación ciudadana conceptos como la conservación y preservación de nuestro patrimonio.

\section{Tabla 8}

Sentido de la iniciativa y espíritu emprendedor

\section{Variable 6}

\begin{tabular}{ccccc}
\hline & Frecuencia & Porcentaje & Porcentaje válido & Porcentaje acumulado \\
\hline 1 & 4 & 1,4 & 1,4 & 1,4 \\
2 & 15 & 5,4 & 5,4 & 6,8 \\
3 & 69 & 24,7 & 24,7 & 31,5 \\
4 & 85 & 30,5 & 30,5 & 62,0 \\
5 & 106 & 38,0 & 38,0 & 100 \\
Total & 279 & 100 & 100 & \\
\hline
\end{tabular}


El sexto ítem analizado en este estudio se corresponde con la competencia del sentido de la iniciativa y el espíritu emprendedor que, pese a su estrecha relación con las Ciencias Sociales puesto que se relaciona con el trabajo en grupo, la búsqueda de información y los contenidos de la geografía humana y económica (Bernal et al., 2014), obtiene unos resultados positivos, aunque algo más discretos: un 68,5\% está de acuerdo o muy de acuerdo, pero hasta un $24,7 \%$ de la muestra responde con dudas sobre la idoneidad de usar el recurso del patrimonio para trabajar esta competencia.

\section{Tabla 9}

Competencia lingüística

\section{Variable 7}

\begin{tabular}{ccccc}
\hline & Frecuencia & Porcentaje & Porcentaje válido & Porcentaje acumulado \\
\hline 1 & 1 & 0,4 & 0,4 & 0,4 \\
2 & 6 & 2,2 & 2,2 & 2,5 \\
3 & 58 & 20,8 & 20,8 & 23,3 \\
4 & 82 & 29,4 & 29,4 & 52,7 \\
5 & 132 & 47,3 & 47,3 & 100 \\
Total & 279 & 100 & 100 & \\
\hline
\end{tabular}

Por último, para finalizar este análisis y discusión de los resultados, también son observables los buenos datos que presenta la variable de la competencia lingüística donde el resultado más elevado es el $47,3 \%$ que se muestra muy de acuerdo, seguido del 29,4\% que también está de acuerdo. Pese a que es una competencia estrechamente relacionada con la enseñanza de la lengua y la literatura, su carácter multidisciplinar provoca que también tenga una buena percepción a la hora de relacionar comunicación lingüística y patrimonio (Gómez-Trigueros \& Ruiz, 2017).

\section{Conclusiones}

Al iniciar este trabajo nos planteábamos como objetivo fundamental el analizar las concepciones del futuro profesorado de Educación Primaria alrededor del uso del patrimonio como recurso para adquirir las competencias clave.

En ese sentido, se partía de una hipótesis inicial, ya sugerida por los estudios de López-Facal et al. (2017) y Arjones-Fernández (2016), que suponía que ciertas materias de Ciencias Sociales están estrechamente ligadas a la adquisición de competencias afines, pero que no se relacionan con el resto de competencias. Algo que concuerda con los estudios empíricos de Cerezo et al. (2020) y con los análisis de Piñana (2019) que comenta la falta de transversalidad en el uso de las competencias clave debido a la falta de un enfoque competencial en nuestro curriculum escolar y a la dificultad del profesorado a la hora de planificar transversalmente los contenidos 
conceptuales (Sánchez, 2017).

Así pues, para conseguir el objetivo específico planteado (O.E. 1), se diseñó y validó un cuestionario como instrumento de investigación con 7 ítems (escala Likert 1-5), que se correspondían con las siete competencias clave que se recogen en el currículum (Real Decreto 126/2014). Dicho instrumento recibió una alta valoración en su fiabilidad y consistencia interna, por lo que fue implementado entre 279 futuros profesores de Primaria.

Con relación al objetivo específico 2, se realizó un análisis estadístico descriptivo de los 7 ítems, reflejando las percepciones que los participantes tienen sobre cómo el patrimonio puede ayudar a conseguir las competencias clave.

A modo de conclusión, es destacable que, tal y como sugería la hipótesis inicial, el uso del patrimonio es percibido como especialmente relevante a la hora de enseñar y aprender las competencias en conciencia y expresiones culturales, así como las sociales y cívicas, que son las dos competencias más ligadas a la asignatura de Ciencias Sociales (Cuenca et al., 2017; Arjones-Fernández, 2016).

Por otro lado, es remarcable que de las siete competencias clave, hay tres que han obtenido una menor puntuación con una media entre 3 y 4 puntos en la escala de valoración, por lo que el alumnado presenta dudas y no percibe tan claramente el patrimonio de utilidad para adquirirlas algo que concuerda con estudios anteriores que recuerdan que la concepción del profesorado sobre competencias clave sigue siendo poco holística (Restrepo, 2017) y que presenta dificultades a la hora de planificar la práctica educativa (Sánchez, 2017): competencia matemática y competencias en ciencia y tecnología, competencia digital y sentido de la iniciativa y espíritu emprendedor.

Sorprenden, pese a los resultados positivos, la menor percepción en los dos últimos casos, debido a las buenas experiencias didácticas existentes a la hora de usar el patrimonio y las TIC (Ibáñez-Etxeberría et al., 2018) o la estrecha relación que hay entre la iniciativa y el espíritu emprendedor con los contenidos de Geografía humana y económica (Bernal et al., 2014).

Esta concepción educativa, muy asentada entre los docentes (Arjones-Fernández, 2016) y entre los futuros docentes como se demuestra en esta investigación, está fundamentada en la propia práctica docente que los participantes observan en el aula, ya que todavía existe una limitación en la planificación educativa que provoca que el profesorado en activo no trabaje las competencias clave de forma holística y transversal (Sánchez, 2017) y que éstas sigan ligadas a materias o áreas de conocimiento determinadas (Restrepo, 2017). Solo la competencia de aprender a aprender y la competencia lingüística tienen una mayor proyección transversal, al menos en lo que corresponde con su trabajo a través del patrimonio.

En definitiva, sería necesaria una mayor presencia de la educación patrimonial en las aulas de Primaria, no solo en el área de Ciencias Sociales, donde sigue siendo escasa (Moreno-Vera \& Alvén, 2020), si no, en otras materias y asignaturas que permitieran un uso y una aproximación más holística del patrimonio y su relación con cualquiera de las competencias clave que el alumnado debe adquirir al finalizar su educación obligatoria. De hecho, el patrimonio se ha revelado como un recurso idóneo para trabajar contenidos conceptuales desde un enfoque competencial (Piñana, 2019) 
puesto que está relacionado con diversas áreas de conocimiento. Aunque, sin embargo, como indica Salido-López (2017) sigue estrechamente ligado, según la normativa a la enseñanza de las Ciencias Sociales y no se aprovecha su carácter holístico, transversal e interdisciplinar, algo que sería especialmente importante de cara a una concepción educativa renovada que forme a los futuros estudiantes para afrontar una sociedad más abierta y más plural (Martín et al., 2021).

\section{Agradecimientos}

Los autores de esta publicación quieren agradecer el apoyo brindado por los respectivos grupos de investigación a los que pertenecen: DICSO de la Universidad de Murcia, cuyos investigadores principales son los doctores Pedro Miralles Martínez y Cosme Jesús Gómez Carrasco, y el grupo IGE de la Universidad de Alicante, cuya investigadora principal es la doctora Rocío Díez Ros. Así mismo, es preceptivo agradecer la ayuda y apoyo de los compañeros y compañeras docentes de la asignatura 17533 Didáctica de las Ciencias Sociales: Historia, en cuyas aulas fue implementado el cuestionario que ha servido como instrumento de investigación en este trabajo. Además, este trabajo es resultado del proyecto de investigación "El pensamiento geográfico e histórico del alumnado de Educación Primaria en la Región de Murcia: propuesta metodológica innovadora para una educación de calidad” (20874/PI/18). Proyecto financiado por la Comunidad Autónoma de la Región de Murcia a través de la convocatoria de Ayudas a proyectos para el desarrollo de investigación científica y técnica por grupos competitivos, incluida en el Programa Regional de Fomento de la Investigación Científica y Técnica (Plan de Actuación 2018) de la Fundación Séneca-Agencia de Ciencia y Tecnología de la Región de Murcia.

\section{Conflicto de intereses}

Los autores declaran no tener ningún conflicto de intereses. Los financiadores no tuvieron ningún papel en el diseño del estudio; en la recopilación, análisis o interpretación de datos; en la redacción del manuscrito, o en la decisión de publicar los resultados.

\section{Contribuciones de los autores}

Conceptualización, Santiago Ponsoda López de Atalaya y Juan Ramón Moreno-Vera; metodología, Juan Ramón Moreno-Vera y Santiago Ponsoda López de Atalaya; software, Juan Ramón Moreno-Vera; validación, Juan Ramón Moreno-Vera; análisis formal, Santiago Ponsoda López de Atalaya; análisis de datos, Santiago Ponsoda López de Atalaya; redacción del borrador original, Juan Ramón Moreno-Vera; redacción, revisión y edición, Juan Ramón Moreno-Vera; adquisición de financiación, Juan Ramón Moreno-Vera.

\section{Referencias}

Arjones-Fernández, M. A. (2016). La Historia del Arte ante el reto del valor social del patrimonio cultural y la competencia para la conciencia y expresiones culturales. En I Congreso Internacional Desigualdad social y educativa en el siglo XXI (1-12). Eumed.

Bernal, A., Delgado, P., y Donoso, M. (2014). Economía del conocimiento, cultura emprendedora y empleabilidad en el ámbito educativo. Una aproximación al caso español. Procedia - Social and behavioral Sciences, 139, 168-174.

Bolívar, A. (2010). Las competencias básicas en el currículo. Ministerio de 
Educación.

Bolívar, A. (2015). Un currículum común consensuado en torno al marco europeo de competencias clave. Un análisis comparativo con el caso francés. Avances en supervisión educativa, 23. https://doi.org/10.23824/ase.v0i23.25

Bustos, A., Castellano, V., Calvo, J., Mesa, R., Quevedo, V., y Aguilar, C. (2019). El aprendizaje basado en retos como propuesta para el desarrollo de las competencias clave. Revista Padres y Maestros, 380. https://doi.org/10.14422/pym.i380.y2019.008

Cerezo, E., Romeu, M. C., y Grao, A. (2020). Key competences and plurilingual education in the Valencian Region (Spain): a curricular analysis of Primary Education. Puls, 43, 35-53.

Colomer, J. C., Sáiz, J., y Bel, J. C. (2018). Competencia digital en futuros docentes de Ciencias Sociales en Educación Primaria: análisis desde el modelo TPACK. Educatio siglo XXI, 36(1), 107-128. https://doi.org/10.6018/i/324191

Cózar, R., y Sáez, J. M. (2017). Realidad aumentada, proyectos en el aula de primaria: experiencias y casos en Ciencias Sociales. EDMETIC, 6(1), 165-180.

Cuenca, J. M., Estepa, J., y Martín-Cáceres, M. (2017). Patrimonio, educación, identidad y ciudadanía. Profesorado y libros de texto en la enseñanza obligatoria. Revista de educación, 375, 136-159. https://doi.org/0.4438/1988-592X-RE-2016-375-338

De la Fuente, A. (2011). Competencias clave en Geografía, Historia y Ciencias Sociales. El busgosu, 6, 36-41.

Egido, I. (2011). Las competencias clave como elemento central del currículo de la enseñanza obligatoria: un repaso a las experiencias europeas. Revista española de educación comparada, 17. 239-262.

Fernández, V. (2005). Finalidades del patrimonio en la educación. Investigación en la escuela, 56, 7-18.

Fontal, O. (2016). El patrimonio a través de la educación artística en la etapa de primaria. Arte, Individuo y Sociedad, 28(1), 105-120. http://dx.doi.org/10.5209/rev ARIS.2016.v28.n1.47683

Fontal, O., Marín, S., y García-Ceballos, S. (2015). Educación de las artes visuales y plásticas en Educación Primaria. Paraninfo.

García-Ceballos, S. (2014). Educación patrimonial, un proyecto de inclusión. Tejuelo: didáctica de la lengua y la literatura, 9, 9-19.

Getsdóttir, S. M., Van, C., y Van, J. (2018). Teaching Historical Thinking and reasoning: construction of an observation instrument. British Educational Research Journal, 44, 960-981. https://doi.org/10.1002/berj.3471

Gómez-Carrasco, C. J. (2013). Los contenidos de ciencias sociales y las capacidades cognitivas en los exámenes de tercer ciclo de educación primaria ¿una evaluación en competencias? Revista complutense de educación, 24(1), 91121

Gómez-Carrasco, C. J., Monteagudo, J., Moreno-Vera, J. R., y Sáinz, M. (2019). Effects of a gamification and flipped-classroom program for teachers in training on motivation and learning perception. Education Sciences, 9(4), 299. https://doi.org/10.3390/educsci9040299 
Gómez-Carrasco, C. J., Monteagudo, J., Moreno-Vera, J. R., y Sáinz, M. (2020). Evaluation of a gamification and flipped-classroom program used in teacher training: perception of learning and outcome. PLoS ONE, 15(10). https://doi.org/10.1371/journal.pone.0241892

Gómez-Redondo, C., Fontal, O., y Ibáñez-Etxeberría, A. (2016). Procesos de patrimonialización e identización patrimonial con uso de TIC en torno al arte contemporáneo. Artnodes, revista de arte, ciencias y tecnología, 17, 52-63

Gómez-Trigueros, I. M., y Ruiz, M. (2017). Análisis de metodologías activas con ABP, transmedia y gamificación para implementar las competencias en Ciencias Sociales y en Literatura. En R. Roig-Vila (ed.) Investigación en docencia universitaria. Diseñando el futuro a partir de la innovación educativa (245-254). Octaedro.

González-Monfort, N. (2019). La educación patrimonial, una cuestión de futuro. Reflexiones sobre el valor del patrimonio para seguir avanzando hacia una ciudadanía crítica. El futuro del pasado, 10, 123-144. https://doi.org/10.14516/fdp.2019.010.001.004

Herrero, C., y Pastor, M. M. (2012). La evaluación continua de las competencias en Ciencias Sociales en el título de Maestro de Educación Primaria. Revista de investigación en educación, 10(1), 30-44.

Ibáñez-Etxeberría, A., Fontal, O., y Rivero, P. (2018). Educación patrimonial y TIC en España: marco normativo, variables estrcuturantes y programas referentes. Arbor, 194(788), a448. https://doi.org/10.3989/arbor.2018.788n2008

Koelher, J., y Mishra, P. (2008). What is technological pedagogical content knowledge (TPACK)? En AACTE Commitee on innovatio and technology (eds.), Handbook of technological pedagogical content Knowledge (TPACK) for educators (1-30). Routledge.

Kortabitarte, A., Gillate, I., Luna, U., y Ibáñez-Etxeberría, A. (2018). Las aplicaciones móviles como recursos de apoyo en el aula de Ciencias Sociales: estudio exploratorio con el app "architecture gothique/romane" en Educación Secundaria. Ensayos, revista de educación de la Facultad de Educación de Albacete, 33(1), 65-79. https://doi.org/10.18239/ensayos.v33i1.1743

López-Facal, R., Gómez-Carrasco, C. J., Miralles Martínez, P., y Prats Cuevas, J. (2017). Educación histórica y desarrollo de competencias En R. López, P. Miralles, J. Prats (dirs.) y C. Gómez (coord.) Enseñanza de la historia y competencias educativas (7-22). Graó.

Martín, A. M., Campos, B., y Pérez, L. (2021). El desarrollo de la profesión docente. Una nueva visión desde la acción pedagógica. UNED.

Moreno-Vera, J. R., y Alvén, F. (2020). Concepts for Historical and geographical Thinking in Sweden's and Spain's Primary Education curricula. Humanities \& Social Sciences Communications, 7(1), 1-10. https://doi.org/10.1057/s41599-020-00601-z

Moreno-Vera, J. R., Ponsoda-López de Atalaya, S., López-Fernández, J. A., y BlanesMora, R. (2020). Holistic or traditional conceptions of heritage among earlychildhood and Primary Trainee Teachers. Sustainability, 12(21), 8921. 
https://doi.org/10.3390/su12218921

Morote, A., y Moreno-Vera, J.R. (2021). La percepción de los futuros docentes de educación secundaria sobre las implicaciones territoriales del cambio climático en destinos turísticos del litoral mediterráneo. Gran Tour, revista de investigaciones turísticas, 23, 261-282.

Najarro, U., y Maroto, J.C. (2019). El desarrollo de las competencias sociales y cívicas en Ciencias Sociales: itinerario didáctico por los restos fenicios de Almuñécar (Granada). Didáctica Geográfica, 20. https://doi.org/10.21138/DG.458

Ortega, D., y Gómez, I.M. (2017). Las webquests y los MOOCs en la enseñanza de las Ciencias Sociales y la formación del profesorado de Educación Primaria. REIFOP, revista electrónica interuniversitaria de Formación del profesorado, 20(2). https://doi.org/10.6018/reifop/20.2.258551

Pagès, J. (2009). Competencia social y ciudadana. Aula de innovación educativa, 187, 7-11.

Pérez, A., Vicente, M., y Hortigüela, D. (2019). ¿Por qué y para qué de las competencias clave en educación física? Análisis de dos posturas contrapuestas. Retos: nuevas tendencias en educación física, deporta y recreación, 35, 7-12

Piñana, E. (2019). La implementación de las competencias clave en Educación Primaria. Un estudio de casos múltiples [Tesis doctoral inédita]. Universidad Autónoma de Madrid.

Real Decreto 126/2014, de 28 de febrero, por el que se establece el currículo básico de la Educación Primaria. (2014). Boletín Oficial del Estado, 52, de 1 de marzo de 2014. $\quad$ https://www.boe.es/buscar/pdf/2014/BOE-A-2014-2222consolidado.pdf

Restrepo, J. (2017). Concepciones sobre competencias matemáticas en profesores de educación básica, media y superior. Boletín REDIPE, 6(2), 104-118. https://revista.redipe.org/index.php/1/article/view/199

Rodríguez-García, A. M., Martínez, N., y Raso, F. (2017). La formación del profesorado en competencia digital: clave para la educación del siglo XXI. Revista Internacional de Didáctica y Organización Educativa, 3(2), 46-65.

Rodríguez-Lestegás, F., Macía, X. C., y Armas, F. X. (2020). De los contenidos a las competencias: aprender transversalmente a través del conocimiento social. Aprendizaxes transversais, 30, 41-56. https://doi.org/10.15304/ie.30.6944

Salido-López, P. V. (2017). La educación artística en el contexto de las competencias clave: del diseño a la evaluación de talleres didácticos en la formación de formadores. Arte, Individuo y Sociedad, 29(2), 349-368.

Sánchez, L. (2017). Las competencias docentes del profesorado de educación secundaria. Importancia percibida e implicaciones en la formación inicial [Tesis de doctorado]. Universitat Jaume I.

Ull, M. Ángeles. (2015). Competencias para la sostenibilidad y competencias en educación para la sostenibilidad en la educación superior. UniPluriversidad, 14(3), 46-58. Recuperado a partir de https://revistas.udea.edu.co/index.php/unip/article/view/21337 
Vázquez-Cano, E. (2016). Dificultades del profesorado para planificar, coordinar y evaluar competencias claves. Un análisis desde la Inspección de Educación. Revista Complutense de Educación, 27(3), 1061-1083. https://doi.org/10.5209/rev RCED.2016.v27.n3.47400 